\title{
Commentary: Can preemptive analgesia decrease opioid use after foregut laparoscopic surgery?
}

\author{
Ivan Azevedo, $\mathrm{MD},{ }^{\mathrm{a}}$ and Paula Ugalde Figueroa, $\mathrm{MD}^{\mathrm{b}}$
}

\footnotetext{
From the a Division of Thoracic Surgery, Department of Oncology Oncobeda, Doctor Beda General Hospital, Rio de Janeiro, Brazil; and ${ }^{\mathrm{b}}$ Department of Pulmonology and Thoracic Surgery, Institut Universitaire de Pneumologie et Cardiologie de Quebec, Quebec City, Quebec, Canada.

Disclosures: Dr Ugalde Figueroa is a speaker for Medtronic, Johnson \& Johnson, and AstraZeneca. Dr Azevedo has nothing to disclose with regard to commercial support.

Received for publication June 18, 2019; accepted for publication June 19, 2019; available ahead of print Aug 1, 2019.

Address for reprints: Paula Ugalde Figueroa, MD, Division of Thoracic Surgery, Institut Universitaire de Cardiologie et de Pneumologie de Quebec, 2725 Chemin Ste-Foy, Quebec City, Quebec G1V 4G5, Canada (E-mail: paula.ugalde@ criucpq.ulaval.ca).

J Thorac Cardiovasc Surg 2020;159:747-8

$0022-5223 / \$ 36.00$

Copyright (C) 2019 by The American Association for Thoracic Surgery

https://doi.org/10.1016/j.jtcvs.2019.06.072
}

For decades, postoperative pain control has been dependent on opioids, which has caused worldwide overprescription at a critical level. ${ }^{1}$ Surgeons are now challenged with how to avoid overprescription of opioids and, above all, the deleterious side effect of chronic opioid dependency.

In this issue of the Journal, Kim and colleagues ${ }^{2}$ attempt to respond this challenge. They retrospectively evaluated the efficacy of a preemptive pain management program in patients who underwent laparoscopic foregut surgery for benign conditions to identify factors associated with the ability to send patients home without an opioid prescription. From 2012 to 2018, Kim and colleagues ${ }^{2}$ implemented, first, an enhanced recovery after surgery (ERAS) protocol, and then, a preemptive pain management protocol at their institution. The 414 patients who underwent laparoscopic foregut surgery during this time were divided into 3 groups for analysis - standard care (control), ERAS protocol only, and ERAS with preemptive pain control. The preemptive strategy consisted of excluding opioids as a default postoperative prescription, administering nonnarcotic pain relievers (eg, acetaminophen and naproxen) according to a schedule to manage pain proactively, and developing an enhanced patient education program directed at partnering with the patient to align expectations for pain management postoperatively and at discharge. Patients in the preemptive pain management group had far fewer opioid prescriptions after surgery $(9.6 \%$ vs $85.2 \%$ vs $87 \% ; P<.001)$, fewer complications $(3.2 \%$ vs $14.8 \%$ vs $15.1 \% ; P=.002)$, and shorter average length of hospital stay $(1.1 \pm 2$ days vs $1.3 \pm 0.7$ days vs $1.8 \pm 1.4$ days; $P<.001)$ than the ERAS-only group and the control group, but they had similar readmission rates within 30 days of surgery. A regression analysis identified factors that contributed to opioid prescriptions after surgery, and in a multivariable model, only the preemptive pain management protocol was associated with a reduction of opioid prescriptions after

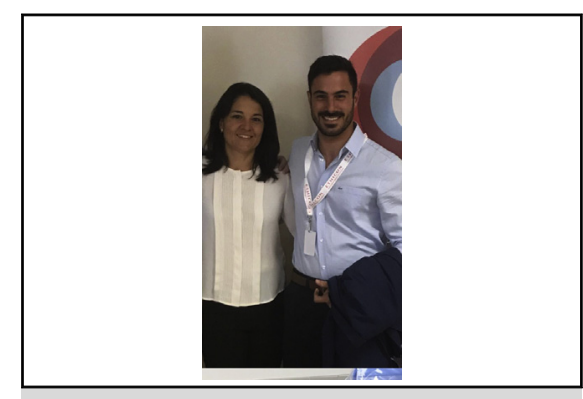

Paula Ugalde Figueroa, MD (left), and Ivan Azevedo, MD (right)

\section{Central Message}

A preemptive pain management program in patients who undergo foregut surgery might decrease the amount of opioids prescribed postoperatively.

See Article page 734 .

surgery. Finally, in a propensity-matched analysis, patients in the preemptive pain management protocol still had significantly fewer opioid prescriptions at discharge, fewer postoperative complications, and a shorter length of hospital stay than the ERAS-alone and control groups. Kim and

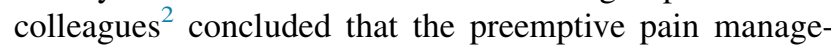
ment program allowed the patients to recover from elective foregut surgery with minimal opioids and facilitated their discharge home without opioids. The adoption of this program could decrease opioid use, opioid availability in the community, and opioid-related deaths.

Implementation of preemptive analgesia, which consists of administering analgesic medication before and during surgery, could eventually decrease hyperalgesia and allodynia by modifying the peripheral and central nervous system's processing of noxious stimuli. ${ }^{3}$ The benefit of preemptive analgesia has been supported by clinical studies with local anesthetics, opioids, and nonsteroidal antiinflammatory drugs. Its clinical impact has remained controversial, likely because of the numerous variables in the studies, such as type of surgery, type of drugs, drug dose, route of administration, treatment duration, and pain assessment. ${ }^{4}$ Large studies that used opioids as a preoperative strategy for preemptive analgesia were not conclusive because of a low level of medical evidence. ${ }^{5}$ Randomized trials have shown that local anesthetic injection around small abdominal incision sites reduces postoperative 
somatic pain but is inadequate for visceral pain. This is one of the reasons that preemptive epidural analgesia is favored in thoracic surgery but not in abdominal surgery. ${ }^{4,6}$

Unfortunately, there are limited data on the use of preemptive analgesia for thoracic and foregut surgery. In principle, it seems reasonable for pain control and without negative side effects in the immediate postoperative period. The adoption and implementation of multimodal analgesic therapies for postoperative pain control after thoracic surgery has been explored by several centers, with satisfactory results for pain control in the immediate postoperative period, specifically during the first 48 hours, but with no difference in the incidence of chronic postthoracotomy pain. ${ }^{7,8}$ The pain management strategies of Kim and colleagues ${ }^{2}$ will likely need modification to decrease opioid use after thoracic surgery, which is associated with more postoperative pain than abdominal surgery, but they offer an interesting starting point.

Assessment of the true value of preemptive analgesia and preemptive patient and physician education requires further research with more comprehensive approaches. ${ }^{9}$ Examining whether opioids were prescribed at discharge or requested later is a good start, but more specific information on the patients' pain profiles-medications administered and postsurgical quality of life-is also needed. Nonetheless, Kim and colleagues ${ }^{2}$ have developed and propose an attractive approach to pain management for patients undergoing foregut surgery.

\section{References}

1. Alam A, Juurlink DN. The prescription opioid epidemic: an overview for anesthesiologists. Can J Anaesth. 2016;63:61-8.

2. Kim MP, Godoy C, Nguyen DT, Meisenbach LM, Chihara R, Chan EY, et al. Preemptive pain-management program is associated with reduction of opioid prescriptions after benign minimally invasive foregut surgery. $J$ Thorac Cardiovasc Surg. 2020;159:734-44.e4.

3. Kelly DJ, Ahmad M, Brull SJ. Preemptive analgesia I: physiological pathways and pharmacological modalities. Can J Anaesth. 2001;48:1000-10.

4. Bong CL, Samuel M, Ng JM, Ip-Yam C. Effects of preemptive epidural analgesia on post-thoracotomy pain. J Cardiothorac Vasc Anesth. 2005;19:786-93.

5. Doleman B, Leonardi-Bee J, Heinink TP, Bhattacharjee D, Llund JN, Williams JP. Pre-emptive and preventive opioids for postoperative pain in adults undergoing all types of surgery. Cochrane Database Syst Rev. 2018;12:CD012624.

6. Leung CC, Chan YM, Ngai SW, Ng KFJ, Tsui SL. Effect of pre-incision skin infiltration on post-hysterectomy pain-a double-blind randomized controlled trial. Anaesth Intensive Care. 2000;28:510-6.

7. Ryu HG, Lee CJ, Kim YT, Bahk JH. Preemptive low-dose epidural ketamine for preventing chronic postthoracotomy pain: a prospective double-blinded, randomized, clinical trial. Clin J Pain. 2011;27:304-8.

8. Matyal R, Montealegre-Gallegos M, Shnider M, Owais K, Sakamuri S, Shakil O, et al. Preemptive ultrasound-guided paravertebral block and immediate postoperative lung function. Gen Thorac Cardiovasc Surg. 2015;63:43-8.

9. Paul A, Afzal M, Bandyopadhyay K, Mishra A, Mookerjee S. Pre-emptive analgesia: recent trends and evidences. Indian J Pain. 2014;27:114. 